\title{
A SIMULATION-BASED ALGORITHM FOR THE INTEGRATED LOCATION AND ROUTING PROBLEM IN URBAN LOGISTICS
}

\author{
Andrés Muñoz-Villamizar \\ Jairo R. Montoya-Torres \\ Escuela de Ciencias Económicas y Administrativas \\ Universidad de La Sabana \\ Chía (Cundinamarca), COLOMBIA
}

\author{
Angel A. Juan \\ José Cáceres-Cruz \\ IN3 - Computer Science Department \\ Open University of Catalonia \\ Barcelona, 08018, SPAIN
}

\begin{abstract}
In most medium and large sized cities around the world, freight transportation operations might have a noticeable impact on urban traffic mobility as well as on city commercial activities. In order to reduce both traffic congestion and pollution levels, several initiatives have been traditionally implemented. One of the most common strategies concerns the allocation of urban distribution warehouses near the city center in order to consolidate freight delivery services. This paper considers the integrated problem of locating distribution centers in urban areas and the corresponding freight distribution (vehicle routing). The combined problem is solved by using a hybrid algorithm which employs Monte Carlo simulation to induce biased randomness into several stages of the optimization procedure. The approach is then validated using real-life data and comparing our results with results from other works already available in the existing literature.
\end{abstract}

\section{INTRODUCTION}

The idea of implementing freight consolidation platforms within urban areas is known in the academic literature as Urban Distribution Centers (UDC) (Taniguchi et al. 1999). The general goal of this research area is to solve - or at least to reduce- traffic problems within urban areas, considering some extra variables like environmental pollution and excessive energy consumption. According to Muñuzuri et al. (2012), this is a critical issue in most large sized European cities. In effect, due to their inherited radial structure these cities tend to show a high concentration of shopping areas, restaurants, and other social attraction poles in the city center, which not only influence mobility and commercial activities but also impose a series of restrictions in flows of freight deliveries. Thus, most urban centers in these cities contain narrow streets with no parking lots or back alleys, which are not well designed to support asymmetric flows of people going to work, shop, eat, or visit tourist attractions (Ligocki and Zonn 1984). In addition, according to several authors (Topp and Pharoah 1994; Muñuzuri et al. 2005; Geroliminis and Daganzo 2006; Delaitre 2008), infrastructure investments in these cities have often been implemented in order to promote environmental sustainability, such as bike lanes, underground and tram systems, more efficient bus systems and the enlargement of pedestrian areas (Daganzo 2010). Despite the clear advantages of these policies, they also led to larger and stricter restrictions regarding freight deliveries.

Among the advantages described by Taniguchi et al. (1999), creating UDCs allows the implementation of a much more efficient urban logistics system, with the same capacity of service than conventional systems but with lower environmental impact. Thus, several cities have decided to put into practice these UDCs in order to take advantage of some of the benefits they offer, including:

- The use of electric vehicles, whose limited autonomy prevents them from travelling long distances. 
- The programming of several successive deliveries or collection routes, which cannot be easily scheduled from outside-urban platforms (Guyon et al. 2012).

- The implementation of cooperative systems for transporting goods (Nemoto 1997, Yang and Odani 2006).

In a previous work, Montoya-Torres, Marquès, and Burlat (2012) proposed a model in which the problems of UDCs location (strategic level), their size (tactical level), and the transportation of goods by assigned vehicles (operating level) are individually addressed following a sequential order. Although this approach allows the decision maker to quickly obtain a reasonably good solution to the integrated problem by sequentially solving each individual sub-problem, the resulting solution may not be globally optimal or even near-optimal. In the logistics literature, it is usual to use the term Location-Routing Problem (LRP) to refer to the combined problems of locating UDCs and routing deliveries. Hence, the three decision levels (strategic, tactical, and operational) can be integrated in a single, yet complex model. This is in fact more challenging as the LRP is known to be a NP-hard optimization problem.

In this paper, an integrated approach to the LRP is proposed. Our algorithm makes use of Monte Carlo simulation in different stages in order to incorporate a biased random behavior to the randomized search process. At different steps of our approach, multi-start phases encapsulate the biasedrandomization of heuristic methods. In fact, each step generates 'promising' sub-solutions in a fast way thanks to the applied non-symmetric probability distribution. The algorithm is then validated using reallife data obtained from Montoya-Torres, Marquès, and Burlat (2012). This data corresponds to the city of Saint-Etienne, in France. The methodology, however, is generic and can be applied to any other city for which proper data is available.

The paper is organized as follows. Section 2 provides more insights about the problem under study. Section 3 is devoted to describe the proposed simulation-based algorithm. Experiments using real-life data are discussed in Section 4. Finally, Section 5 presents some concluding remarks and opportunities for further research.

\section{PROBLEM STATEMENT}

Urban Distribution Centers are public terminals with complex installations and multiple functions, including transshipment yards, stores, wholesale markets, information centers, exhibition halls and meeting rooms, etc. (Taniguchi et al. 1999). These UDCs are designed to meet the diverse needs of an urban logistics system through the use of advanced information systems. With these advanced information systems, optimization models, and heuristic algorithms can be developed to: (a) determine efficient transportation routes and programming systems; and (b) reduce the number of trucks that are required to provide a given level of service to customers.

The UDCs can also help small and medium-sized enterprises to carry out efficient transportation of goods through the mechanization and automation of the handling of the goods. These terminals can also facilitate the implementation of cooperative freight transportation systems, which allow to share and absorb the costs generated by the distribution of products, thus reducing the costs of collection and/or delivery of goods and the costs of acquisition and maintenance of vehicles and necessary facilities for the operation. Figure 1 shows a schematic representation of an urban distribution center. As stated by Taniguchi et al. (1995), truck traffic can be reduced by adopting cooperative freight systems (Figure 2). This reduction of vehicles not only leads to a monetary savings for businesses, but also relieves the city vehicular level, reduces the environmental impact (fewer pollutants) and eventually improves the life quality of the citizens.

The problems of locating, sizing, and operating of the urban centers of distribution can be viewed as a Location-Routing Problem. The LRP seeks to locate facilities within a set of possible destinations and design routes between customers assigned to them, allowing, as a result, to determine the capacity or size of the facility and, if is necessary, of the vehicles. 
- Delivery points (shops, etc.)
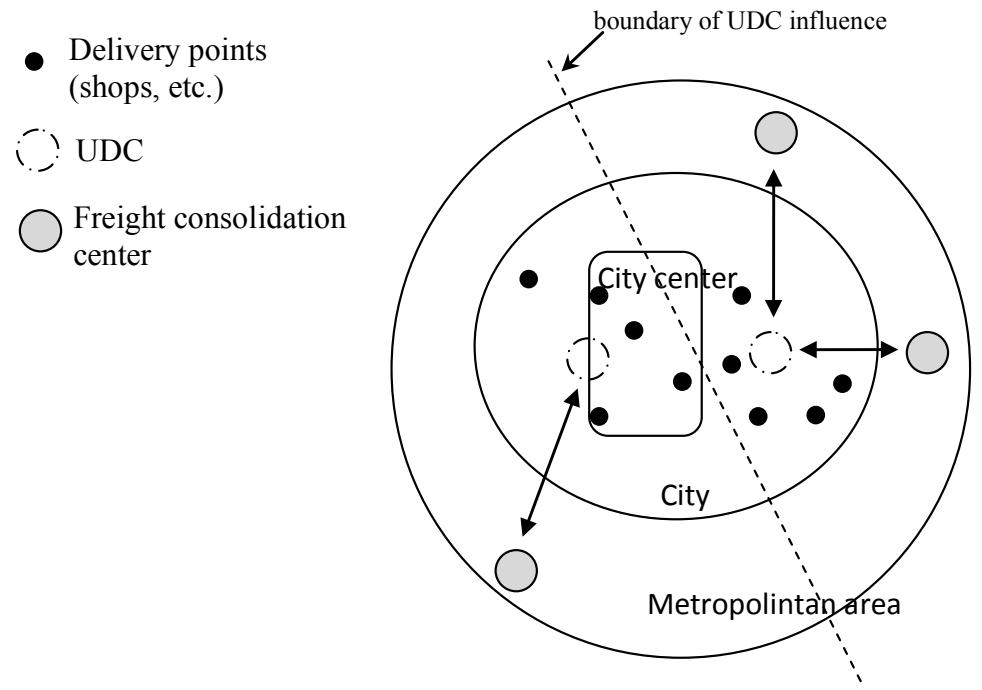

Figure 1: Schematic representation of location of UDC (Montoya-Torres, Marquès, and Burlat 2012).

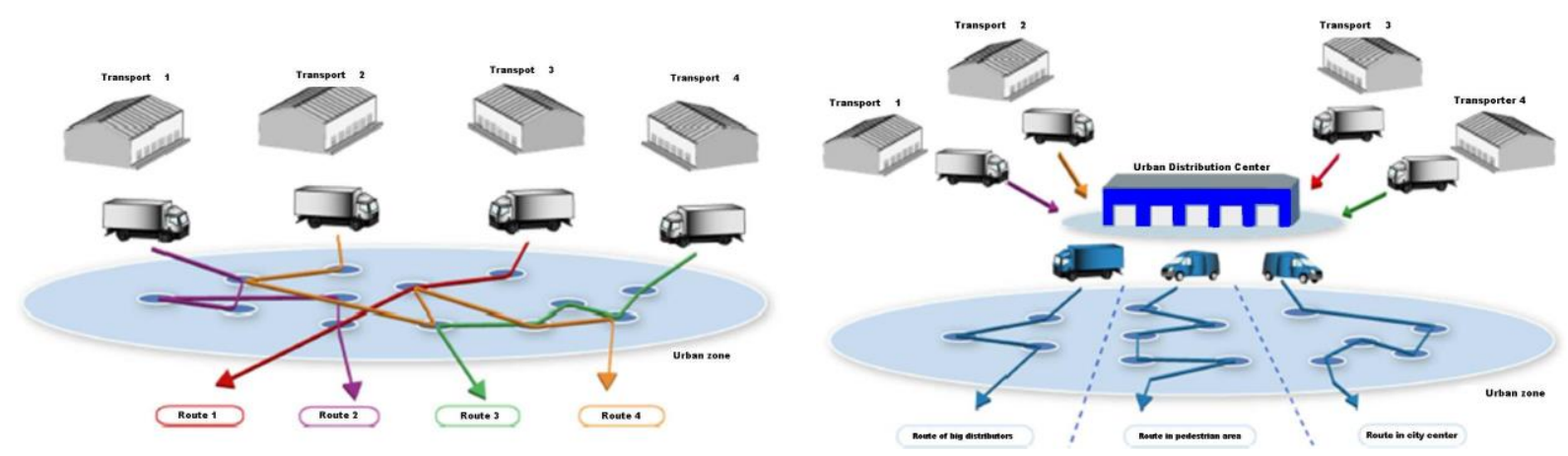

Figure 2: Comparison of distribution modes using traditional routing versus UDC.

The LRP is conceptually more difficult than the classical Facility Location Problem (FLP), and also than the classical Vehicle Routing Problem (VRP). Both problems can be seen as special cases of the LRP. If all clients are visited directly from a depot, the LRP becomes a FLP. Likewise, if the depot location is fixed, the LRP becomes a VRP (Nagy and Salhi 2007). Thus, the LRP is a NP-hard problem, since it comprises the FLP and VRP, which are known to be NP-hard. Nagy and Salhi (2007) classify heuristics to solve LRPs in:

a) Sequential methods that solve first the location problem and then the routing problem. The concept of sequential solution does not allow feedback between both phases. Srivastava and Benton (1990) noted that these methods are able to provide good quality solutions that can serve as a benchmark for other heuristics.

b) Methods based on clustering that first divide customers into groups, then assign a facility to each of these groups, and finally solve the associated VRP.

c) Iterative methods, which iteratively handle location and routing phases, i.e.: they decompose the problem into two sub-problems and then use the solution from one to feed the other in a recurrent and alternate way. 
Muñoz-Villamizar, Montoya-Torres, Juan, and Cáceres-Cruz

d) Hierarchical methods, which first solve the location problem and then the routing problem.

\section{OUR APPROACH}

The LRP is a problem that consists of three sub-problems: (a) the location of depots; (b) the assignment or sizing of clients to responsible depots; and (c) the general vehicle routing problem. To simultaneously deal with these subproblems, the method proposed in this paper uses a two-stage strategy: first, it deals with the problem of location; then, it solves an integrated allocation and vehicle routing problem. The details of each of the two parts of our approach are described next. At each step, the randomized criteria is used which mainly consists on the biasedrandomized selection of the elements in a ranked list, then a different promising output is likely to be created each time the entire procedure is executed. Using a biased probabilistic distribution, the elements at the top of the list receive more probabilities of being selected than those at the bottom of the list, but potentially all elements could be selected. The whole process is shown in Figure 3.

\subsection{Depot Location}

The final objective of a location problem is to determine the location of a number of depots that have to be open among a set of potential candidate sites, in order to minimize the total costs associated. We propose a stochastic heuristic which makes use of Monte Carlo simulation to perform a biased randomization clustering. This clustering process uses the concept of center of mass for grouping nodes. The purpose of this heuristic is to create the input for the next algorithm step which solve, in an integrated manner, the allocation of clients to depots and the associated vehicle routing.

The heuristic procedure for the depot location is explained as follows. First, the customers center of mass is obtained, considering their demands. A stopping criterion is defined -e.g., how many different "locations" are requested. Next, the area where customers are distributed is divided from the center of mass and then the clients are allocated to these sub-areas, forming clusters. The number of clusters depends on the number of depots to be located. The randomization of this division is ensured by rotating the base axis for the partition through a uniform random value between $[0,2 \pi)$ divided by the number of clusters. Next, centers of mass are calculated for each cluster according to the customers allocated. For each cluster, a sorted list is generated according to the distances between its centers of mass and the location of depots. Using a geometric probability distribution, a depot is randomly assigned to each cluster. This stochastic procedure is repeated over and over until the termination criterion is met.

\subsection{Assignment and Vehicle Routing}

Once the location of depots has been obtained, the LRP becomes a multi-depot vehicle routing problem (MDVRP). For that reason, the proposed algorithm employs the logic behind the procedure proposed by Juan et al. (2012). This algorithm uses a multi-start procedure to address the integrated problem of allocation and vehicle routing. Once all routes are found, this algorithm uses a simulation-based method to improve those routes without changing the allocation of customers to each depot. This simulation-based method is a variant of the SR-GCWS and SR-GCWS-CS algorithms proposed, respectively, in Juan et al. $(2010,2011)$. An interesting feature of this algorithm is that it uses Monte Carlo simulation to generate random-variates from a Geometric probability distribution. These randomvariates are then used to induce a biased-randomization process in the well-known Clarke \& Wright (1964) savings (CWS) constructive heuristic for the VRP.

The procedure for solving the assignment and routing is as follows. First an ordered list for each pool is computed using the distance to each customer. Then, a biased randomization is performed using a geometric distribution in order to randomly assign clients to each depot according to the ordered list of distances between depots and customers. By assigning each customer to a single depot, an allocation map is randomly generated. Using this map and the capacity constraints-, the routing problem of each depot-customers set is solved by applying the CWS heuristic. This generates a global initial solution. This procedure is iterated a number of time until the stopping condition is met. This stopping condition is usually given by the maximum time the stochastic algorithm is allowed to be executed, which is a user-defined parameter. The best solution found during the multi-start process is then improved with a variant of the aforementioned SR-GCWS algorithm. In this step, the solutions are evaluated separately for each depot seeking to improve their routes without changing the allocation of customers. So, this last algorithm solves the different VRP instances -one for each depot- that are part of the MDVRP instance. The algorithm takes the execution time as stopping condition. Finally, the best LRP solution found is recorded. 


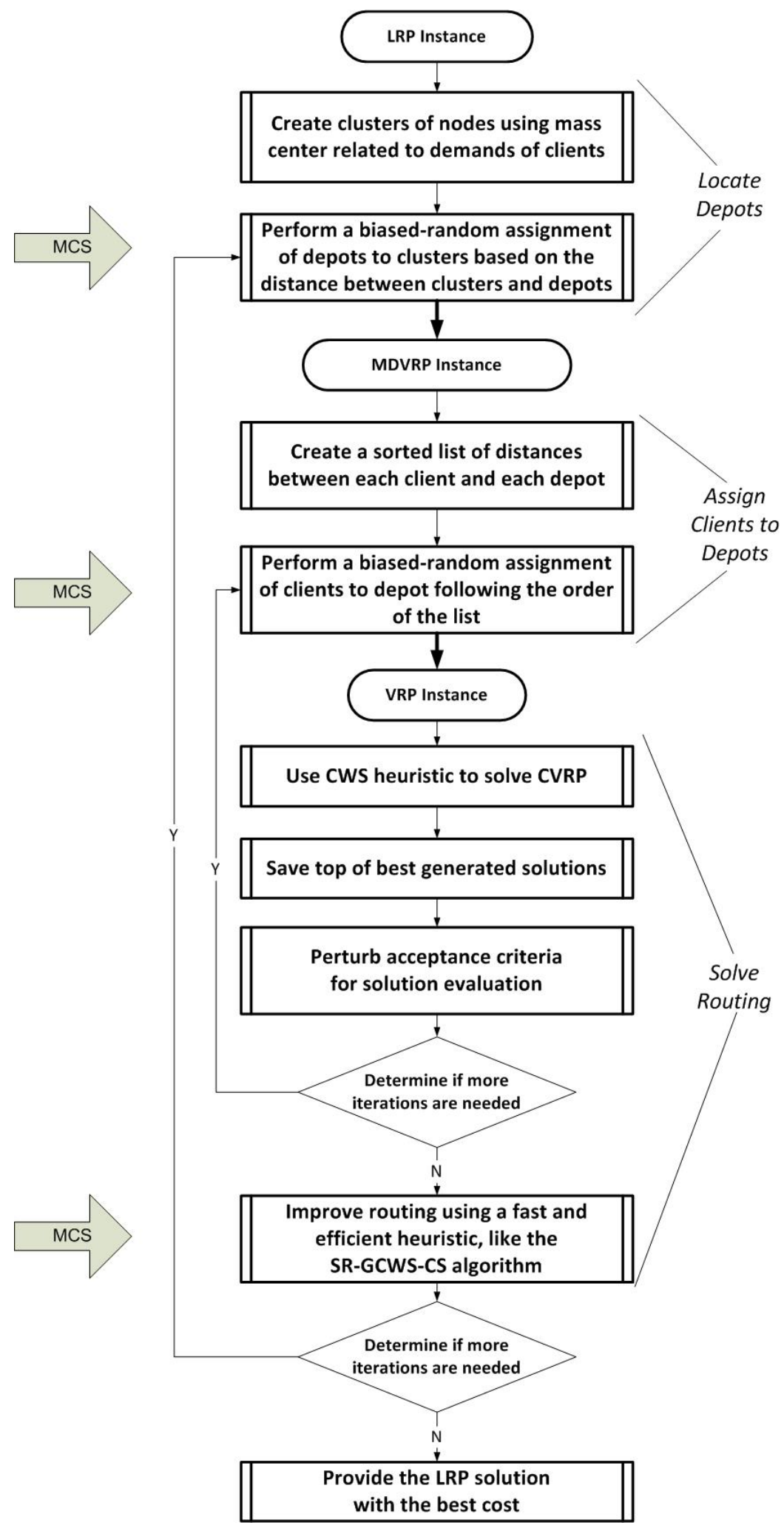

Figure 3: Flowchart of our approach. 


\section{NUMERICAL EXPERIMENTS}

This experimental section is focused on analyzing the efficiency of the proposed approach by comparing the solutions it generates with the solutions provided by an exact methodology developed in Montoya-Torres, Marquès, and Burlat (2012). In our experiments, we used the real data provided by the aforementioned authors, which corresponds to the French city of Saint-Etienne. This case study suggests the location of an Urban Distribution Center for the central area of the city, consisting of 1,227 stores. The exact model designed for this situation gradually raised the number of customers to be served, from 20 to up to 100 customers. Given the complexity of the problem, for a larger number of customers the exact model becomes inefficient. Given the characteristics of this case study, where only one depot should be located, the complexity of the problem is reduced to a VRP once the location of the depot is determined.

\subsection{Scenario A: 20 Clients and 20 Candidate Locations for One Single Depot}

In order to test our approach in a small-size scenario, where the solutions provided by the exact approach are expected to be optimal, we first used an instance with just 20 customers. This instance was solved with both the exact and the simulation-based approaches for three different cases: considering one, two, and three routes. In this instance, every customer is a possible location of the distribution center. Customers' demands and vehicle load capacity are deterministic and known. Table 1 presents the results obtained using both approaches for each case. Notice that our simulation-based approach was able to match the solutions provided by the exact method. Hence, we can state a preliminary good performance of the proposed method, at least for small instances. Figure 4 shows the location of customers, and the solutions for one, two, and three routes.

Table 1: Results for Scenario A with 20 clients and one, two and three routes.

\begin{tabular}{|l|c|c|c|}
\hline \multicolumn{1}{|c|}{ Case } & Exact method & Simulation-based approach & Gap \\
\hline One Route & $\$ 2,696$ & $\$ 2,696$ & $0.0 \%$ \\
\hline Two Routes & $\$ 2,834$ & $\$ 2,834$ & $0.0 \%$ \\
\hline Three Routes & $\$ 3,246$ & $\$ 3,246$ & $0.0 \%$ \\
\hline
\end{tabular}
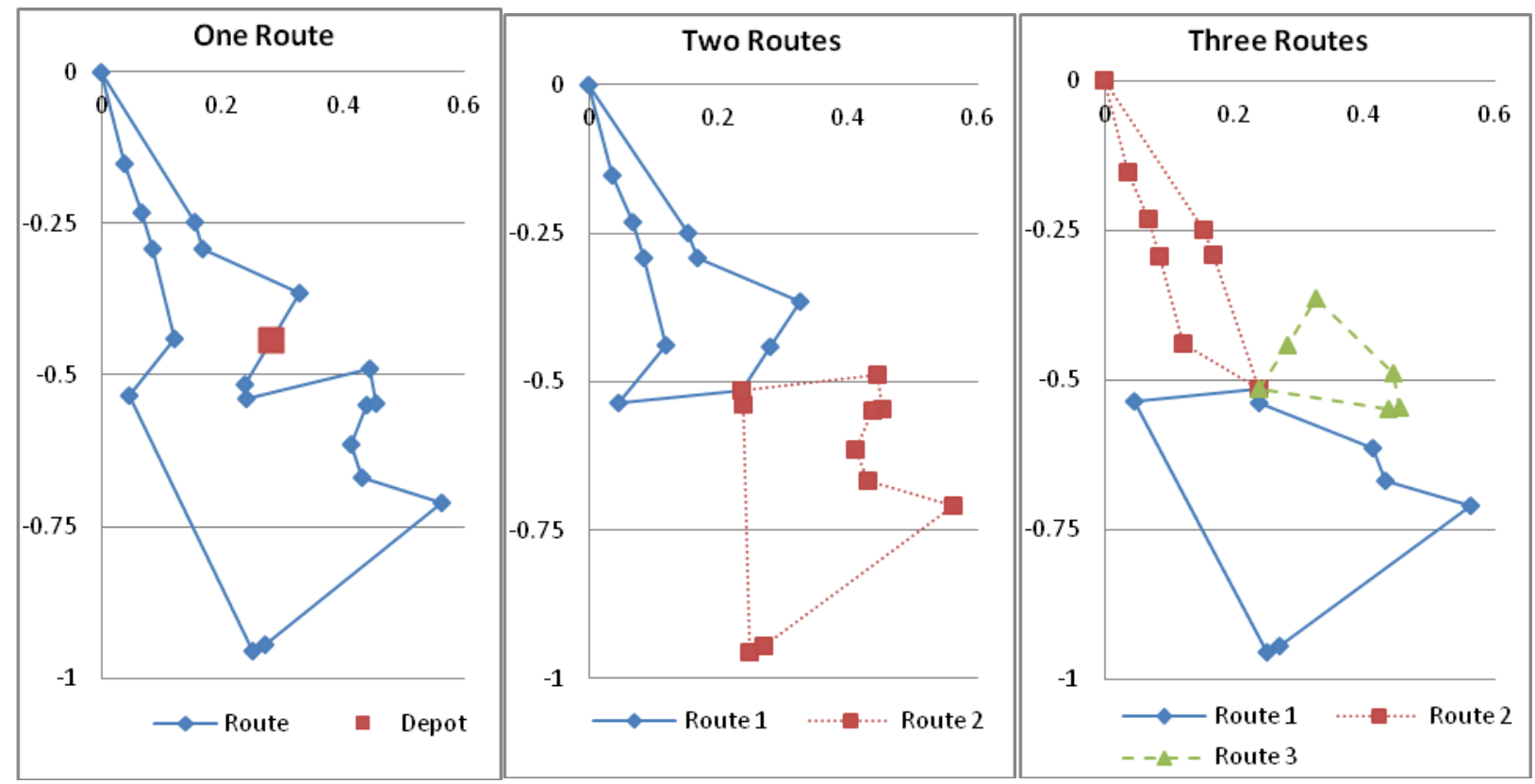

Figure 4: Solutions obtained for Scenario A with 20 clients and one, two, and three routes. 


\subsection{Scenario B: 50 Clients and 8 Candidate Locations for One Single Depot}

A second, medium-size scenario with 50 customers is proposed. The number of possible locations of the depot is now reduced to 8 specific points. In this case, the customer and potential depots locations are defined. The solutions were defined using only 3 routes. Table 2 presents the associated costs for this 50customers instance as generated for each methodology. Notice that the exact method is not very efficient for this problem size and the result presented in the table corresponds to the best solution found -not the optimal one- after running the model for several hours running the model. In this case, our simulationbased method produces a much better solution (-12.34\% of gap). Figure 5 shows the solutions generated by each method.

Table 2: Results for Scenario B with 50 customers and three routes.

\begin{tabular}{|c|c|c|c|}
\hline Exact Method & Our Approach & Cost Gap & \% Gap \\
\hline$\$ 6,264$ & $\$ 5,491$ & $-\$ 773$ & $-12.34 \%$ \\
\hline
\end{tabular}
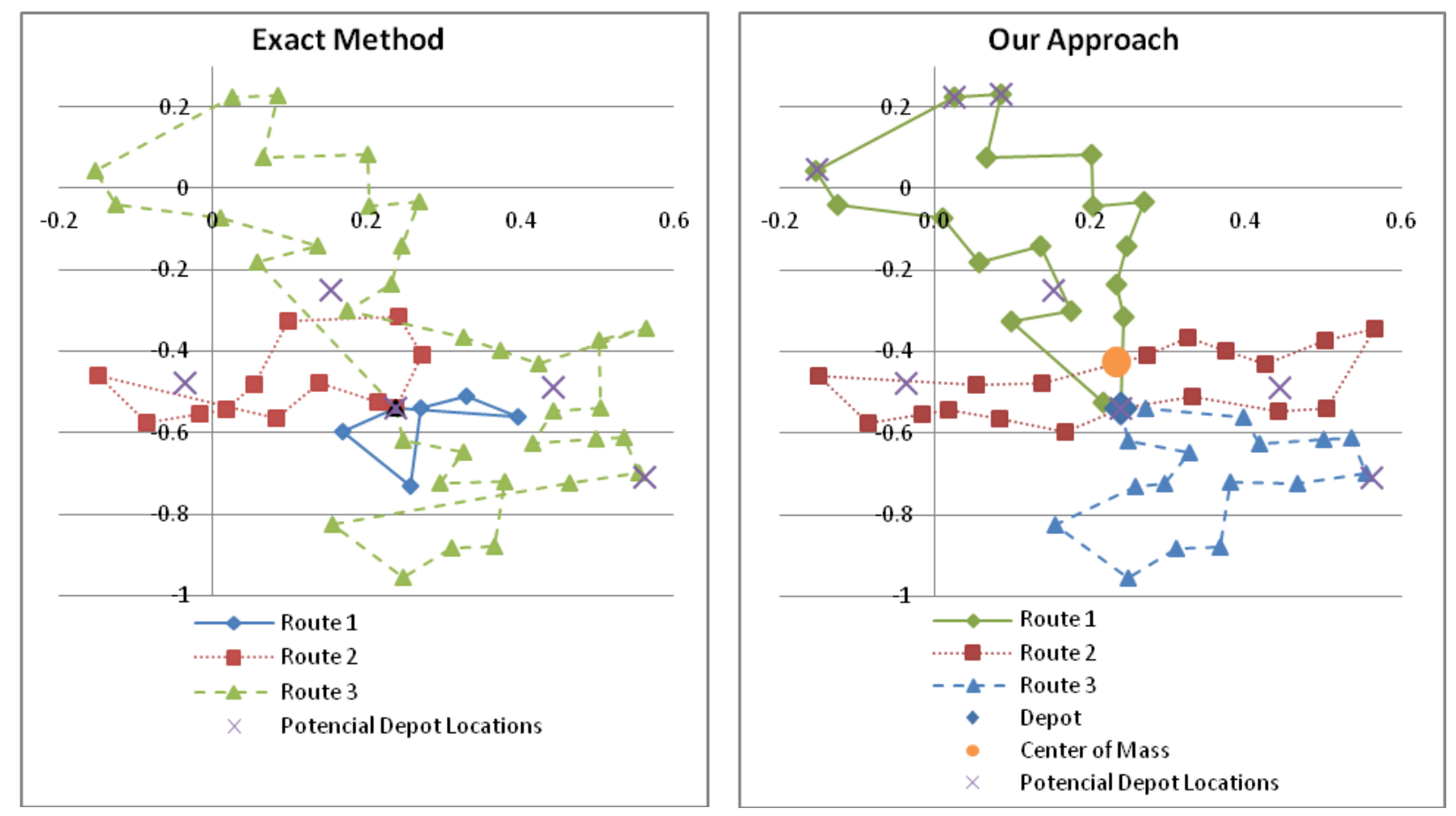

Figure 5: Solutions obtained for Scenario B with 50 customers using both methods.

\subsection{Scenario C: Depot at the Periphery}

Finally, a third test scenario is proposed to evaluate our approach. While in the previous scenario the depot location was centered, in this new scenario this location is in the urban periphery. Table 3 shows a comparison between the best solution obtained using our simulation-based algorithm for both cases -i.e. centered depot vs. periphery depot. From the results, we can state that an urban distribution center should be close to the customers center of mass-i.e. within the city center and not in the periphery. Figure 6 shows both solutions. 
Table 3: Results for Scenario C with 50 clients, three routes and the depot located at the periphery.

\begin{tabular}{|c|c|c|c|}
\hline $\begin{array}{c}\text { Solution } \\
\text { Centered-Depot }\end{array}$ & $\begin{array}{c}\text { Solution } \\
\text { Periphery-Depot }\end{array}$ & Cost Gap & \% Gap \\
\hline$\$ 5,491$ & $\$ 7,400$ & $\$ 1,909$ & $34.77 \%$ \\
\hline
\end{tabular}

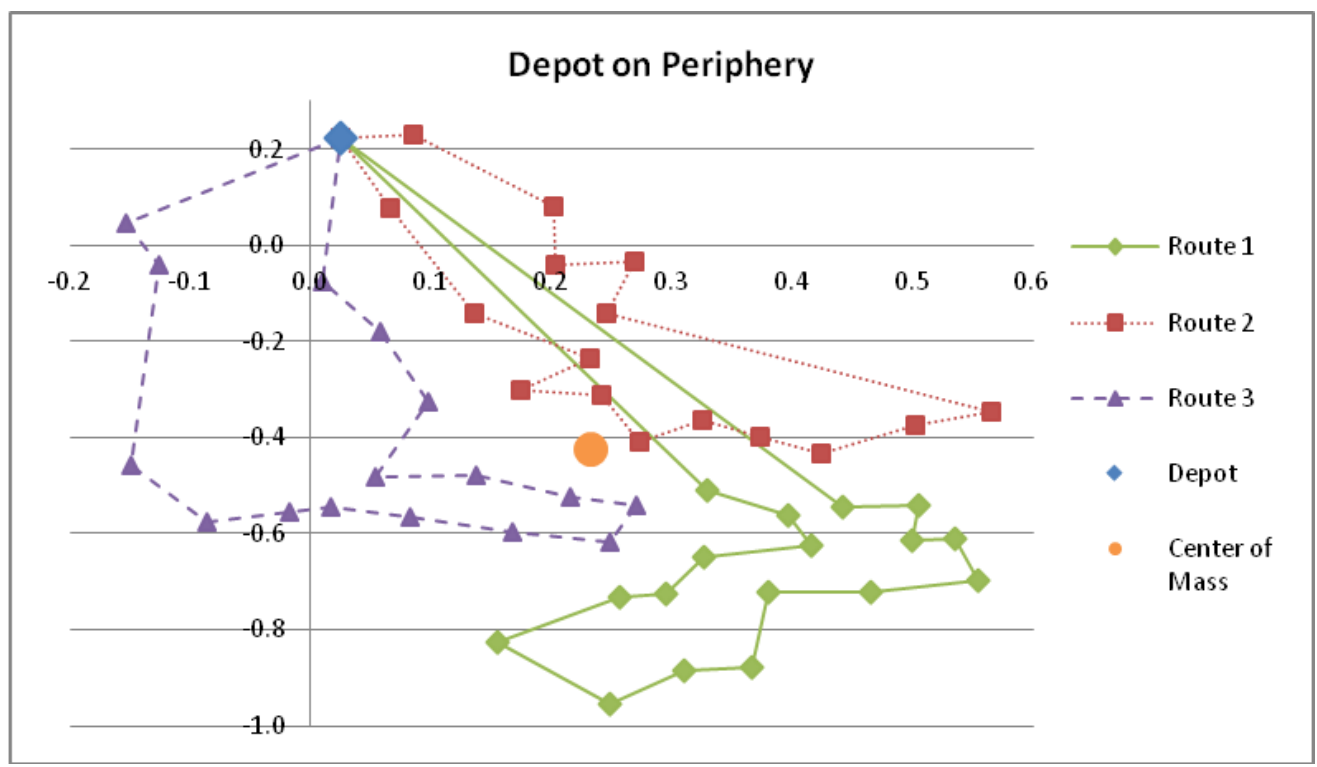

Figure 6: Solution obtained for Scenario C with 50 clients and one depot located at the periphery.

\section{CONCLUSIONS AND PERSPECTIVES}

This paper has discussed the combined Location Routing Problem (LRP) in urban scenarios. The LRP is a NP-hard combinatorial optimization problem with relevant applications to mobility and traffic congestion in medium and large sized cities. The idea of using urban distribution centers for freight transportation within a city emerges as a solution to reduce carbon emissions, traffic and noise contamination in downtown. The approach presented in this paper considered in an integrated way the problems of locating distribution centers in urban areas, their dimensioning, and the corresponding freight distribution. To solve this problem, we have proposed a simulation-optimization approach which employs Monte Carlo simulation to add biased random behavior to different heuristic procedures in order to efficiently search for a near-optimal solution. Our approach has been compared against an exact method using real data already available in the literature. According to the obtained results, our simulation-based algorithm seems to perform quite well in small size scenarios and is even able to outperform the exact method in medium and large size scenarios, where the complexity of the problem makes the exact method inefficient. For further research, interesting opportunities emerge when other objective functions regarding environmental or social impact evaluations are considered. In fact, others probabilistic distributions can be used for studying its impact on results. Also it can be combined with other decisions variables, like the stochastic demands (Juan et al. 2013; Caceres-Cruz et al. 2012) in order to address more realistic-complex scenarios. This may allow to a multi-objective or multi-criteria decision making problem. This type of decision problems could be of interest for public-policies decision makers.

\section{ACKNOWLEDGMENTS}

This work has been partially supported by the Ibero-American Program for Science, Technology and Development (CYTED2010-511RT0419, IN3-HAROSA network) and by the Spanish Ministry of Science 
and Innovation (TRA2010-21644-C03). This paper was written while the second author (Prof. Jairo R. Montoya-Torres) was Visiting Scholar at Leeds University Business School, University of Leeds, UK, supported by a Marie Curie International Incoming Fellowship within the 7th European Community Framework Programme (project "DISRUPT", grant No. ESR- 299255).

\section{REFERENCES}

Caceres-Cruz, J., S. Grasman, A. Juan, T. Bektas and J. Faulin. 2012. "Combining Monte-Carlo Simulation with Heuristics for Solving the Inventory Routing Problem with Stochastic Demands." In Proceedings of the 2012 Winter Simulation Conference, edited by C. Laroque, J. Himmelspach, R. Pasupathy, O. Rose, and A.M. Uhrmacher, 3114-3122. Piscataway, New Jersey: Institute of Electrical and Electronics Engineers, Inc.

Clarke, G., and J. Wright. 1964. "Scheduling of Vehicles from a central Depot to a Number of Delivering Points." Operations Research 12:568-581.

Daganzo, C.F. 2010. "Structure of competitive transit networks." Transportation Research Part B 44:434-446.

Delaître, L. 2008. Méthodologie pour optimiser le transport de marchandises en ville, application aux villes moyennes et dans le cadre de l'agglomération de La Rochelle. Thèse de doctorat, Ecole Nationale Supérieure des Mines de Paris, France.

Geroliminis, N., and C.F. Daganzo. 2006. "A Review of Green Logistics Schemes Used in Cities around the World." Working paper UCB-ITS-VWP-2005-5, UCB Center for Future Urban Transport: A Volvo Center of Excellence, Institute of Transport Studies, University of California Berkeley, USA. http://www.metrans.org/nuf/documents/geroliminis.pdf [Accessed: November 26, 2011].

Guyon, O., N. Absi, D. Feillet, and T. Garaix. 2012. "A Modeling Approach for Locating Logistics Platforms for Fast Parcel Delivery in Urban Areas." Procedia - Social and Behavioral Sciences 39:360368.

Juan, A., B. Barrios, M. Coccola, S. Gonzalez, J. Faulin, and T. Bektas. 2012. "Combining biased randomization with meta-heuristics for solving the multi-depot vehicle routing problem." In Proceedings of the 2012 Winter Simulation Conference, edited by C. Laroque, J. Himmelspach, R. Pasupathy, O. Rose, and A.M. Uhrmacher, Poster. Piscataway, New Jersey: Institute of Electrical and Electronics Engineers, Inc. http://informs-sim.org/wsc12papers/includes/files/pos120.pdf [Accessed: April 1, 2013]

Juan, A., J. Faulin, J. Jorba, D. Riera, D. Masip, and B. Barrios. 2011. "On the Use of Monte Carlo Simulation, Cache and Splitting Techniques to Improve the Clarke and Wright Savings Heuristics." Journal of the Operational Research Society 62:1085-1097.

Juan, A., J. Faulin, J. Jorba, J. Caceres, and J. Marques. 2013. "Using Parallel \& Distributed Computing for Solving Real-time Vehicle Routing Problems with Stochastic Demands." Annals of Operations Research 207:43-65.

Juan, A., J. Faulin, R. Ruíz, B. Barrios, and S. Caballe. 2010. "The SR-GCWS Hybrid Algorithm for Solving the Capacitated Vehicle Routing Problem." Applied Soft Computing 10:215-224.

Ligocki, C., and L.E. Zonn. 1984. "Parking Problems in Central Business Districts." Cities 1:350-355.

Montoya-Torres, J.R., G. Marquès, and P. Burlat. 2012. "A conceptual framework for location, sizing and operation of urban distribution centers with sustainable performance metrics." In Proceedings of the 4th International Conference on Information Systems, Logistics and Supply Chain, Québec, Canada, August 26-29.

Muñuzuri J., J. Larrañeta, L. Onieva, and P. Cortés. 2005. "Solutions Applicable by Local Administrations for Urban Logistics Improvement." Cities 22:15-28.

Muñuzuri J., P. Cortés, J. Guadix, and L. Onieva. 2012. "City Logistics in Spain: Why It Might Never Work." Cities 29: 133-141. 
Nagy, G., and S. Salhi. 2007. "Location-routing: Issues, Models and Methods." European Journal of Operational Research 177:649-672.

Nemoto T. 1997. "Area-wide Inter-carrier Consolidation of Freight in Urban Areas.” Transport Logistics $1: 87-103$.

Srivastava, R., and W.C. Benton. 1990. "The Location-routing Problem: Considerations in Physical Distribution System Design.” Computers \& Operations Research 17:427-435.

Taniguchi, E., M. Noritake, T. Yamada, and T. Izumitani. 1999. "Optimal Size and Location Planning of Public Logistics Terminals." Transportation Research Part E 35:207-222.

Taniguchi, E., T. Yamada, and T. Yanagisawa. 1995. "Issues and Views on Cooperative Freight Transportation Systems." In: 7th World Conference on Transport Research.

Topp, H., and T. Pharoah. 1994. "Car-free City Centres." Transportation 21:231-247.

Yang, D., and M. Odani. 2006. "Analysis on Movement of Profit for the Partner Companies in Jointdelivery System.” Proceedings of Infrastructure Planning 33:327.

\section{AUTHOR BIOGRAPHIES}

ANDRÉS MUÑOZ VILLAMIZAR is a Research Assistant within the School of Economics and Management Sciences at Universidad de La Sabana, Chia, Colombia. He is pursuing the Master in Operations Management program at the same university, and holds the Industrial Engineering degree from Escuela Colombiana de Ingeniería Julio Garavito, Bogotá, Colombia. His research has been focused on solving location-routing problems found in the configuration of urban logistic platforms. He can be contacted by email to the address andresmuvi@unisabana.edu.co.

JAIRO R. MONTOYA-TORRES is Full Professor in the School of Economics and Management Sciences at Universidad de La Sabana, Chia, Colombia. He is currently Visiting Scholar (2013-2015) at the Leeds University Business School at University of Leeds, UK, under a Marie Curie International Incoming Fellowship. His research interests lie broadly in simulation and optimisation of logistics and production systems, scheduling, and supply chain management under collaborative and sustainable environments. He is a member of several academic societies, including ACM and EUROSIS, and has served as Guest Editor or within the Editorial Board of various academic journals. His email address is jairo.montoya@unisabana.edu.co and his web page is http://jrmontoya.wordpress.com.

ANGEL A. JUAN is an Associate Professor of Applied Optimization and Simulation in the Computer Science Department at the IN3-Open University of Catalonia (Barcelona, Spain). Dr. Juan holds a Ph.D. in Applied Computational Mathematics, an M.S. in Information Systems and Technology, and an M.S. in Applied Mathematics. His research interests include Applied Optimization and Randomized Algorithms. He has published over 140 peer-reviewed papers in international journals, books, and proceedings. Currently, he is the coordinator of the CYTED-IN3-HAROSA@IB Network and an editorial board member of both the International Journal of Data Analysis Techniques and Strategies and the International Journal of Information Systems and Social Change. His e-mail is ajuanp@gmail.com and his web page is http://ajuanp.wordpress.com.

JOSE CACERES-CRUZ is a Ph.D. student of the Information and Knowledge Society Doctoral Programme in the Open University of Catalonia, Barcelona, Spain. He holds a Specialization on Information Systems from the Central University of Venezuela and a Computer Engineering degree from the Simón Bolívar University. His main research interests are related to optimization of vehicle routing on realistic scenarios, and simulation solutions. His email address is jcaceres@uoc.edu. 